\title{
INTERFERÊNCIA DO FUNGO ENTOMOPATOGÊNICO Beauveria bassiana (VUIL.) NO DESENVOLVIMENTO DO PREDADOR Podisus nigrispinus EM LABORATÓRIO
}

\section{INFLUENCE OF THE ENTOMOPATHOGENIC FUNGUS Beauveria bassiana (VUIL.) ON DEVELOPMENT OF THE PREDATOR Podisus nigrispinus IN LABORATORY}

\author{
Anderson Mathias HOLTZ ${ }^{1,2}$ \\ Ricardo Antonio POLANCZYK ${ }^{1,3}$ \\ José Salazar ZANUNCIO Junior ${ }^{1,4}$ \\ Dirceu PRATISSOLI, ${ }^{1,3}$ \\ Renata Aparecida FERREIRA ${ }^{5}$ \\ Leandro Pin DALVI ${ }^{6,7}$ \\ Franciene de Campos BORTOLINI ${ }^{5}$
}

\begin{abstract}
RESUMO
O predador Podisus nigrispinus (Dallas) (Heteroptera: Pentatomidae) e o fungo entomopatogênico Beauveria bassiana (Bals.) Vuill. (Hypocreales: Clavicipitaceae) são importantes agentes de controle biológico de lepidópteros. Em programas de manejo integrado de pragas, técnicas podem ser utilizadas conjuntamente para reduzir a população da praga. Porém, a eficácia de um organismo benéfico pode ser afetada, negativamente, por outro inimigo natural. Por isso, avaliou-se o efeito do entomopatógeno Beauveria bassiana sobre o potencial reprodutivo e crescimento populacional de $P$. nigrispinus através dos parâmetros da tabela de vida de fertilidade. O fungo $B$. bassiana foi inoculado sobre o predador ou sua presa Tenebrio molitor L. (Coleóptera: Tenebrionidae), avaliando os efeitos direto e indireto do contato. A taxa líquida de reprodução (Ro) foi de 24,9 e 34,5 com a aplicação do fungo sobre a presa e sobre o predador, respectivamente, contra 52,5 e 72,7 no controle. A taxa intrínseca de crescimento populacional $(\mathrm{rm})$ foi de 0,08 e 0,09 no tratamento com $B$. bassiana sobre a presa e sobre o próprio predador e de 0,09 e 0,11 no controle. A razão finita de aumento $(\lambda)$ foi de 1,08 e 1,09 quando o predador teve contato com fungo e de 1,10 e 1,11 no controle. $O$ crescimento populacional de $P$. nigrispinus foi maior sem a presença de $B$. bassiana, demonstrando que a associação desse fungo juntamente com $P$. nigrispinus pode diminuir a eficácia do predador no controle de pragas.
\end{abstract}

Palavras-chave: Tabela de vida; Asopinae; controle microbiano; Beauveria bassiana.

\begin{abstract}
The predator Podisus nigrispinus (Dallas) (Heteroptera: Pentatomidae) and the entomopathogenic fungus Beauveria bassiana (Bals.) Vuill. (Hypocreales: Clavicipitaceae) are important biological control agents of Lepidoptera. In integrated pest management programs, techniques can be used together to reduce the pest population. However, the effectiveness of a beneficial organism can be negatively affected by another natural enemy. Therefore, was evaluated the effect of the entomopathogen $B$. bassiana on reproductive potential and the population growth of $P$. nigrispinus, with the parameters of the fertility life table. The fungus $B$. bassiana was inoculated on the predator or on its prey Tenebrio molitor (Coleoptera: Tenebrionidae), evaluating direct and indirect effects by contact. The net reproductive rate (Ro) was 24.9 and 34.5 with the application of the pathogen on the prey and on the predator, respectively, against 52.5 and 72.7 in the control. The intrinsic rate of increase $\left(r_{m}\right)$ was 0.08 and 0.09 in with $B$. bassiana on the prey and on the predator and 0.09 and 0.11 in the control. The finite rate of increase $(\lambda)$ was 1.08 and 1.09 if the predator had contact with the pathogen and of 1.10 and 1.11 in the control. The population growth of $P$. nigrispinus was better without the presence of the fungus, showing that the association between different agents of biological control can reduce the efficiency in the pest control.
\end{abstract}

Key-words: Life table; Asopinae; microbial control; Beauveria bassiana.

\footnotetext{
1 Doutor em Entomologia.

2 Instituto Federal de Educação, Ciência e Tecnologia do Espírito Santo - Campus Alegre, Professor, Alegre, Espírito Santo, Brasil. E-mail: aholtz@bol.com.br.

${ }^{3}$ Departamento de Produção Vegetal da Universidade Federal do Espírito Santo, Professor adjunto, Pesquisador CNPq. Alegre, Espírito Santo, Brasil. E-mail: polanczyk@cca.ufes.br; pratissoli@cca.ufes.br.

Movimento de Educação Promocional do Espirito Santo-MEPES, Professor, Alegre, Espírito Santo, Brasil. E-mail: jjzanuncio@insecta.ufv.br.

Graduando, Universidade Federal do Espírito Santo, Alegre, Espírito Santo, Brasil. E-mail: retarracha@hotmail.com; fran.bortolini@hotmail.com.

${ }^{6}$ Doutorando, Departamento de Fitotecnia da Universidade Federal de Viçosa, Av. PH Rolfs s/n - Campus Universitário, CEP: 36570 000,

Viçosa, Minas Gerais, Brasil. E-mail: leandropin@yahoo.com.br.

${ }^{7}$ Autor responsável para correspondência
} 
HOLTZ, A.M. et al. Interferência do fungo entomopatogênico Beauveria bassiana...

\section{INTRODUÇÃO}

O setor industrial de base florestal tem sido marcado pela utilização crescente de madeiras provenientes de reflorestamento, colocando o Brasil em destaque mundial, incentivando a implantação de florestas renováveis e a preservação das naturais. Neste cenário, as espécies do gênero Eucalyptus, Myrtaceae exótica proveniente da Austrália destacam-se como as mais utilizadas, movimentando todos os setores da indústria madeireira (Zanuncio et al., 1992; Iwakiri et al., 2000).

A expansão dos plantios de eucalipto, com extensas áreas de monocultivo proporcionou a ocorrência de pragas, que têm provocado sérios prejuízos aos povoamentos florestais. No Brasil, há relatos de insetos das ordens Lepidoptera, Coleoptera e Isoptera provenientes de mirtáceas nativas, como a goiabeira, danificando plantas de eucalipto (Bragança et al., 1998). Somente da ordem Lepidoptera foram relatadas mais de 280 espécies nativas alimentado-se de plantas de eucalipto, sendo a espécie Thyrinteina arnobia (Stoll) (Lepidoptera: Geometridae) a mais importante praga desfolhadora dessa essência florestal no Brasil (Santos et al., 2000).

O predador Podisus nigrispinus (Dallas) (Heteroptera: Pentatomidae) vem sendo utilizado contra lepidópteros desfolhadores em grandes plantios de eucalipto (Torres et al., 2006), por sua facilidade de criação massal, ocorrência nos mais variados ecossistemas brasileiros e eficiência no controle de pragas por ser um predador generalista (Zanuncio et al., 1994). No entanto, o controle biológico deve ser implementado em conjunto com outras táticas, de modo a incrementar os fatores de mortalidade natural no ecossistema, as quais são selecionadas de acordo com parâmetros técnicos, econômicos, ecológicos e sociais (Dent, 2000).

Os métodos de controle biológico são claramente importantes para determinados insetospraga, sendo um componente essencial e amplamente sugerido como base no Manejo Integrado de Pragas (Dent, 2000). Dessa forma, os inseticidas biológicos, tais como as formulações a partir do fungo entomopatogênico Beauveria bassiana (Bals.) Vuill. (Hypocreales: Clavicipitaceae) podem fornecer uma alternativa valiosa aos inseticidas sintéticos que possuem algumas desvantagens particularmente relacionadas aos perigos ambientais e os danos provocados à saúde humana (Glare \& O'CallaGhan, 2000).

A interação de bioinseticidas à base de fungo com $P$. nigrispinus, visando à supressão de espécies praga, pode ser extremamente valiosa como componente do manejo integrado de pragas, uma vez que, os bioinseticidas a base de fungo apresentam largo espectro no controle de diversas pragas (Faria \& Magalhães, 2001). No entanto, são necessários estudos específicos para determinar as possíveis interações decorrentes dessa associação, visto que, efeitos negativos de entomopatógenos sobre insetos benéficos, já foram registrados (Santos et al., 2006).

Desta forma, essa pesquisa objetivou avaliar a interferência do fungo entomopatogênico $B$. bassiana sobre o predador $P$. nigrispinus através dos parâmetros da tabela de vida de fertilidade.

\section{MATERIAL E MÉTODOS}

O experimento foi conduzido no laboratório de Entomologia do Núcleo de Desenvolvimento Científico e Tecnológico em Manejo Fitossanitário de Pragas e Doenças (NUDEMAFI), do Centro de Ciências Agrárias da Universidade Federal do Espírito Santo (CCA-UFES), em Alegre, ES, em sala climatizada com temperatura de $25 \pm 1{ }^{\circ} \mathrm{C}$, umidade relativa de $70 \pm 10 \%$ e fotofase de $14 \mathrm{~h}$.

Ninfas de primeiro estádio de $P$. nigrispinus foram obtidas da criação massal do laboratório de Entomologia do NUDEMAFI. Essas ninfas foram criadas, sob as mesmas condições laboratoriais, do primeiro estádio à fase adulta em grupos de cinco em placas de Petri $(15,0 \times 1,2 \mathrm{~cm})$ contendo um chumaço de algodão no seu interior, sendo diariamente umedecido com água destilada. A alimentação desses insetos foi realizada em dieta com pupas de Tenebrio molitor L. (Coleoptera: Tenebrionidae) (Zanuncio et al., 1994).

No início da fase adulta, os predadores foram pesados em balança com precisão de 0,1 $\mathrm{mg}$, e sexados pela aparência externa da genitália e tamanho do corpo. Esses indivíduos de $P$. nigrispinus foram acasalados e distribuídos em copos plásticos de $500 \mathrm{~cm}^{3}$ (um casal por copo), contendo papel absorvente em seu interior como substrato para oviposição e tubos de $2,5 \mathrm{~cm}^{3}$, do tipo anestésico, para fornecimento de água (Pires et al., 2006; Zanuncio et al., 2008). Os percevejos receberam como alimento pupas de $T$. molitor (Zanuncio et al., 2001).

Foram utilizados 80 casais recém emergidos à fase adulta do predador, que foram divididos em quatro tratamentos: água destilada pulverizada sobre os predadores; água destilada pulverizada sobre a presa (antes de serem oferecidas ao predador) e; suspensão do bioinseticida Boveril $^{\circledR}$, aplicada diretamente sobre o predador e sobre a presa na concentração $10^{8}$ conídios $\mathrm{cm}^{-3}$, comumente utilizada em testes de patogenicidade.

A tabela de vida de fertilidade foi estimada calculando-se os seguintes parâmetros de crescimento populacional do predador $P$. nigrispinus: taxa líquida de reprodução (Ro), que corresponde ao número de descendentes fêmeas produzidas por uma única fêmea durante seu período de vida; a razão finita de aumento $(\lambda)$ definida como o número de vezes que a população se multiplica; a taxa intrínseca de crescimento populacional $\left(r_{\mathrm{m}}\right)$, que relaciona a taxa de aumento populacional por unidade de tempo; o tempo de geração $(T)$, que equivale ao período de tempo entre o nascimento dos pais com o nascimento da nova geração e o tempo para duplicar o número de 
HOLTZ, A.M. et al. Interferência do fungo entomopatogênico Beauveria bassiana...

fêmeas (DT), que determina o tempo necessário para dobrar a população (Krebs, 1994).

Todos os parâmetros da tabela de vida foram estimados e analisados utilizando o programa LIFETABLE.SAS escrito por Maia et al. (2000) o qual permite a comparação de pares de tratamentos empregando-se o teste t.

\section{RESULTADOS E DISCUSSÃO}

A exposição ao fungo $B$. bassiana afetou o crescimento populacional de $P$. nigrispinus nas condições experimentais adotadas. A taxa líquida de reprodução (Ro) foi de 72,7 e 52,5 descendentes fêmeas contra 34,5 quando $P$. nigrispinus teve contato direto e 24,9 no contato indireto com o fungo $B$. bassiana (Tabela 1), mostrando que, provavelmente, houve um efeito negativo sobre o predador, tanto pelo contato direto, quanto pelo contato indireto com o fungo. Moreira et al. (1995) estudando o crescimento populacional do predador Tynacantha marginata Dallas (Heteroptera: Pentatomidae), sobre pupas de $T$. molitor, encontraram resultados semelhantes de taxa liquida de reprodução (50,7 descendentes fêmeas) aos de $P$. nigrispinus criado sem a presença do fungo. Porém, quando há associação do fungo $B$. bassiana a taxa de crescimento reduz, ficando semelhante aos resultados de crescimento populacional de outros heterópteros predadores como Supputius cincticeps (Stal) (Assis Júnior. et al., 1998), demonstrando que estes valores mudam conforme a espécie de predador, alimentação e a interação dos mesmos com outros agentes de controle biológico, como os fungos entomopatogênicos.

TABELA 1 - Parâmetros da tabela de vida de fertilidade de Podisus nigrispinus exposto direta e indiretamente ao entomopatógeno Beauveria bassiana.

\begin{tabular}{lccccc}
\hline Tratamentos & Ro & T & rm & $\lambda$ & DT \\
\hline Predador + água & $72,7 \pm 9,6 \mathrm{a}$ & $40,6 \pm 1,3 \mathrm{a}$ & $0,11 \pm 0,0 \mathrm{a}$ & $1,11 \pm 0,0 \mathrm{a}$ & $6,6 \pm 0,2 \mathrm{a}$ \\
Presa + água & $52,5 \pm 7,2 \mathrm{~b}$ & $40,8 \pm 1,9 \mathrm{a}$ & $0,10 \pm 0,0 \mathrm{a}$ & $1,10 \pm 0,0 \mathrm{a}$ & $7,1 \pm 0,4 \mathrm{a}$ \\
Predador + fungo & $34,5 \pm 8,5 \mathrm{c}$ & $41,7 \pm 2,0 \mathrm{a}$ & $0,09 \pm 0,0 \mathrm{~b}$ & $1,09 \pm 0,0 \mathrm{~b}$ & $8,1 \pm 0,5 \mathrm{~b}$ \\
Presa + fungo & $24,9 \pm 5,3 \mathrm{c}$ & $40,8 \pm 3,4 \mathrm{a}$ & $0,08 \pm 0,0 \mathrm{~b}$ & $1,08 \pm 0,0 \mathrm{~b}$ & $8,7 \pm 0,7 \mathrm{~b}$
\end{tabular}

Médias seguidas da mesma letra nas colunas não diferem entre si pelo teste t, ao nível de 5\% de significância. Ro: taxa líquida de reprodução. T: tempo de geração. rm: taxa intrínseca de crescimento populacional. $\lambda$ : razão finita de aumento.DT: tempo para duplicar a população.

O tempo de geração $(T)$ da população foi semelhante entre os indivíduos de $P$. nigrispinus independentemente de terem sido expostos a $B$. bassiana (Tabela 1). Valores semelhantes foram encontrados para a mesma espécie de predador alimentado com pupas de T. molitor em plantas de algodão ou em plantas daninhas (35,4 dias) (Oliveira et al., 2002; Evangelista et al., 2003) e para Podisus maculiventris (Say) em dieta artificial (32,55 a 40,64) (Wittmeyer \& Coudron, 2001), indicando que o tempo de desenvolvimento de $P$. nigrispinus não é afetado pelo fungo entomopatogênico $B$. bassiana. Provavelmente, a reação alcalina ou ácida do conteúdo intestinal dos insetos pode ser uma das causas de eliminação de muitos microrganismos potencialmente patogênicos (Alves, 1998), o que poderá ter minimizado a ação desses fungos sobre desenvolvimento de $P$. nigrispinus.

A taxa intrínseca de crescimento populacional $(\mathrm{rm})$ foi menor nos tratamentos que o predador $P$. nigrispinus teve contato com o fungo em relação aos tratamentos que não foi usado $B$. bassiana (Tabela 1), demonstrando que, possivelmente, houve um efeito negativo sobre o predador, tanto pelo contato direto, quanto pelo contato indireto com o fungo. Entretanto, esta taxa de crescimento foi maior $(0,08$ a 0,11$)$ (Tabela 1$)$, mesmo na presença do fungo, do que para indivíduos da espécie $S$. cincticeps $(0,05)$ alimentados apenas com pupas de T. molitor (Assis Júnior et al., 1998). Porém, esses valores de crescimento populacional foram menores aos de $T$. marginata $(0,39)$ criados sobre pupas de $T$. molitor e plantas de eucalipto (Moreira et al., 1995), semelhantes aos de $P$. nigrispinus $(0,13)$ criado em plantas de algodão e plantas daninhas (Evangelista et al., 2003) e semelhantes aos de $P$. maculiventris $(0,05$ a 0,11$)$ criado em dieta artificial (Wittmeyer \& Coudron, 2001). Estes resultados indicam que $P$. nigrispinus apresenta maior número de descendentes fêmeas durante uma geração que $S$. cincticeps, menor que $T$. marginata e semelhante para $P$. maculiventris e para a mesma espécie de predador criada em plantas de algodão e daninha. Porém, com a associação com o fungo $B$. bassiana observa-se que houve uma diminuição nos valores da taxa intrínseca de crescimento em relação aos predadores $P$. maculiventris e $P$. nigrispinus criados em plantas de algodão e erva daninha. Sendo assim, o contato direto ou indireto com o fungo afeta negativamente o predador $P$. nigrispinus, pois este pode atuar de forma deletéria sobre os predadores pela inviabilização de ovos, larvas e adultos, alteração do ciclo de vida e dificuldades no encontro da presa (Magalhães et al., 1998).

A razão finita de aumento populacional $(\lambda)$ do predador sem tratamento foi maior do que indivíduos expostos ao fungo (Tabela 1). Porém, mesmo na presença do fungo, a razão finita de 
HOLTZ, A.M. et al. Interferência do fungo entomopatogênico Beauveria bassiana...

aumento populacional apresentou valores próximos $(1,11$ e 1,08) (Tabela 1) aos do predador $S$. cincticeps $(1,03$ e 1,05) também criado em pupas de T. molitor (Assis Jr. et al., 1998). No entanto, esses valores foram menores do que aqueles obtidos para o predador T. marginata $(1,48)$ criado com T. molitor em plantas de eucalipto (Moreira et al., 1995). Os resultados demonstram que a associação entre o predador $P$. nigrispinus com o fungo $B$. bassiana pode afetar negativamente a razão finita de aumento populacional $(\lambda)$ e, consequentemente, reduzir o número de gerações seguintes do predador em campo.

O tempo para a população dobrar em número (DT) também foi afetado negativamente pelo tratamento com fungo. O DT foi aumentado dois dias quando comparado com o predador que não teve contato com $B$. bassiana (Tabela 1$)$. Porém, o tempo de duplicação da população $(6,6$ e $8,7)$ foi maior do que o encontrado por Wittmeyer \& Coudron (2001) para $P$. maculiventris $(0,19$ a 0,99$)$. Estes resultados indicam que o predador $P$. nigrispinus, quando em contato com o fungo entomopatogênico, sofre efeitos negativos para a manutenção e desenvolvimento de sua população, efeito este que provavelmente se repetira em campo. Segundo Alves (1998) e Magalhães et al.,
(1998), o contato entre predadores e entomopatógenos, em condições de campo ou em laboratório, ocorre quando os predadores são atingidos diretamente durante as aplicações do fungo, quando entram em contato com plantas contaminadas ou quando se alimentam de insetos já infectados, e este contato pode ter efeito deletério sobre a população de predadores causando mortalidades ou queda na reprodução.

A fertilidade específica $\left(m_{x}\right)$ de fêmeas do predador $P$. nigrispinus sem aplicação do fungo foi maior ao longo da vida reprodutiva, com maior área sob a curva do que as fêmeas do predador que receberam a aplicação de $B$. bassiana (Figura 1a). $A$ fase adulta de insetos predadores é marcada por um período sem reprodução, seguido da fase reprodutiva, apresentando, posteriormente, um esforço reprodutivo máximo, declinando rapidamente com o envelhecimento das fêmeas (Rabinovich, 1978). O ponto máximo de reprodução e declínio reprodutivo de $P$. nigrispinus ocorreu entre $\circ 35^{\circ}$ e $\circ 70^{\circ}$ dia após a emergência dos adultos (Figura 1), independente dos tratamentos. Estes valores foram próximos aos observados em $S$. cincticeps criados com T. molitor e suplemento vegetal, apresentando picos de produção entre o $45^{\circ}$ e o $75^{\circ}$ dia (Assis Júnior et al., 1998).
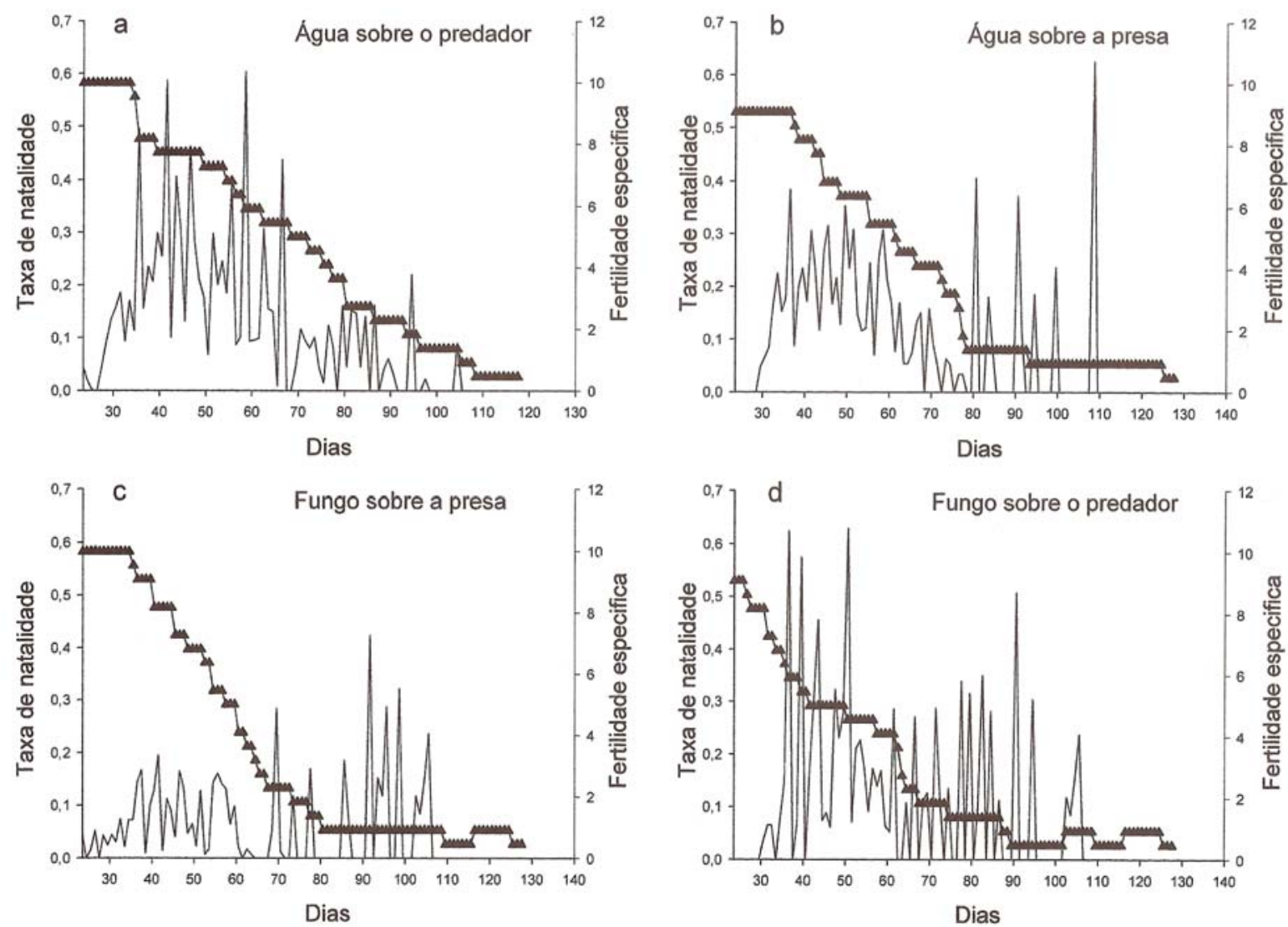

$\longrightarrow$ Taxa de natalidade $\left(I_{x}\right)$ Fertilidade especifica $\left(m_{x}\right)$

FIGURA 1 - Taxa de natalidade e fertilidade específica de Podisus nigrispinus com aplicação do fungo entomopatogênico Beauveria bassiana sobre a presa Tenebrio molitor e sobre o predador. 
HOLTZ, A.M. et al. Interferência do fungo entomopatogênico Beauveria bassiana...

A associação direta entre o fungo e o predador proporcionou uma curva de fertilidade especifica de $P$. nigrispinus mais desuniforme, apresentando baixa taxa de fertilidade até o $65^{\circ}$ dia e picos intercalados do $69^{\circ}$ até $108^{\circ}$ dia (Figura 1d), demonstrando que o fungo interferiu negativamente na taxa de reprodução de $P$. nigrispinus quando comparado com o tratamento que não houve associação entre o fungo e o predador. No tratamento em que o predador foi associado indiretamente com o $B$. bassiana (presa + fungo), a fase reprodutiva também apresentou picos desuniformes, reforçando a hipótese que o fungo afeta negativamente a fertilidade especifica do predador. Entretanto estes tratamentos não ocasionaram mortalidade até 10 dias após a inoculação do fungo. França et al. (2006) observaram efeitos negativos de Metarhizium anisopliae (Metsch.) Sorokin e B. bassiana quando aplicados sobre 0 percevejo predador $P$. nigrispinus, demonstrando que fungos entomopatogênicos podem provocar a mortalidade e queda na reprodução do predador (Fernandes et al., 1985; La Rosa, 2000).

A taxa de natalidade apresentou declínio mais brusco com aplicação direta do fungo $B$. Bassiana sobre $P$. nigrispinus. Aos 50 dias a taxa foi em torno de $40 \%$, para contato indireto, contra
$25 \%$ quando o predador teve contato direto com o fungo (Figura 1c-d). Segundo Alves (1998) os fungos entomopatogênicos têm um mecanismo de ação rápida para infectar e colonizar o hospedeiro. Esta ação é caracterizada pela capacidade de penetração, que pode ser via oral, pelos espiráculos e pelo tegumento, provocando a rápida mortalidade do seu hospedeiro.

\section{CONCLUSÕES}

Em laboratório, $B$. bassiana interferiu negativamente no desenvolvimento de $P$. nigrispinus, tanto quando houve aplicação direta sobre o predador como quando houve aplicação sobre a presa alternativa T. molitor.

Estudos mais amplos devem ser realizados para utilização simultânea de $B$. bassiana e $P$. nigrispinus no controle biológico de lepidópteros desfolhadores em plantios de eucalipto.

\section{AGRADECIMENTOS}

$$
\text { Ao Conselho Nacional de }
$$

Desenvolvimento Científico e Tecnológico (CNPq) e à Fundação de Amparo a Pesquisa do Estado do Espírito Santo (FAPES) pela concessão de bolsas.

\section{REFERÊNCIAS}

1. ALVES, S. B. Fungos entomopatogênicos. In: ALVES, S. B. (Ed.). Controle microbiano de insetos. 2. ed. Piracicaba: FEALQ. 1998. p. 289-381.

2. ASSIS JUNIOR et al. Efeito da suplementação de folhas de Eucalyptus urophylla no desenvolvimento e reprodução do predador Supputius cincticeps (Stäl) (Heteroptera: Pentatomidae). Anais da Sociedade Entomológica do Brasil, v. 27, n. 2, p. 245-253, 1998.

3. BRAGANCA, M. A. L. et al. Effects of environmental heterogeneity on Lepidoptera and Hymenoptera populations in Eucalyptus plantations in Brazil. Forest Ecology and Management, v. 103, n. 2-3, p. 287-292, 1998.

4. DENT, D. Insect pest management. 2. ed. Cambridge: Cabi Bioscience, 2000. 410 p.

5. EVANGELISTA, W. S. J. et al. Efeito de plantas daninhas e do algodoeiro no desenvolvimento, reprodução e preferência para Oviposição de Podisus nigrispinus (Dallas) (Heteroptera: Pentatomidae). Neotropical Entomology, v. 32, n. 4, p. 677-684, 2003.

6. FARIA, M. R.; MAGALHÃES, B. P. O uso de fungos entomopatogênicos no Brasil. Biotecnologia Ciência \& Desenvolvimento, v. 22, n. 1, p. 18-21, 2001.

7. FERNANDES, P. M.; LECUONA, R. E.; ALVES, S. B. Patogenicidade de Beauveria bassiana (Bals.) Vuill à broca-docafé Hypothenemus hampei (Ferrari 1867) (Coleoptera: Scolytidae). Ecossistema, v. 10, n. 2, p. 176-182, 1985.

8. FRANÇA, I. W. B. et al. Efeito de Metarhizium anisopliae (Mestsch) Sorok. e Beauveria bassiana (Bals) Vuill. sobre o percevejo predador Podisus nigrispinus (Dallas) (Hemiptera: Pentatomidae). Neotropical Entomology, v. 35, n. 3, p. 349-356, 2006.

9. GLARE, T. R.; O'CALLAGHAN, M. Bacillus thuringiensis: biology, ecology and safety. Chichester: John Wiley, 2000 $350 \mathrm{p}$.

10. IWAKIRI, S. et al. Resíduos de serrarias na produção de painéis de madeira aglomerada de eucalipto. Scientia Agraria, v. 1, n. 1-2, p. 23-28, 2000.

11. KREBS C. J. Ecology: the experimental analysis of distribution and abundance. 4. ed. New York: Harper Collins Col. Publication, 1994. $801 \mathrm{p}$.

12. LA ROSA, W. et al. Effect of Beauveria bassiana and Metarhizium anisopliae (Deuteromycetes) upon the coffee berry borer (Coleoptera: Scolytidae) under field conditions. Journal of Economic Entomology, v. 93, n. 5, p. 1409-1414 2000.

13. MAGALHÃES, B. P.; MONNERAT, R.; ALVES, S. B. Entomopatógenos. In: ALVES, S.B. (Ed.). Controle microbiano de insetos. 2. ed. Piracicaba: FEALQ, 1998. p. 207-210.

14. MAIA, A. H. N.; LUIZ, A. J. B.; CAMPANHOLA, C. Statistical inference on associated fertility life table parameters using Jackknife technique: computational aspects. Journal of Economics Entomology, v. 93, n. 2, p. 511-518, 2000.

15. MOREIRA, L. A. et al. Tabelas de fertilidade e de esperança de vida de Tynacantha marginata Dallas (Heteroptera, Pentatomidae, Asopinae) alimentado com larvas de Tenebrio molitor L. (Coleoptera, Tenebrionidae) e folhas de Eucalyptus urophylla. S.T. Blake. Revista Brasileira de Zoologia, v. 12, n. 2, p. 255-261, 1995.

16. OLIVEIRA, J. E. M. et al. Efeito das plantas do algodoeiro e do tomateiro, como complemento alimentar, no desenvolvimento e na reprodução do predador Podisus nigrispinus (Dallas) (Heteroptera: Pentatomidae). Neotropical Entomology, v. 31, n. 1, p. 101-108, 2002. 
HOLTZ, A.M. et al. Interferência do fungo entomopatogênico Beauveria bassiana...

17. PIRES, E. M. et al. Potencial reprodutivo horário do predador de lagartas desfolhadoras do eucalipto: Podisus nigrispinus (Heteroptera: Pentatomidae). Revista Árvore, v. 30, n. 6, p. 1039-1044, 2006.

18. RABINOVICH, J. E. Ecologia de poblaciones animales. Washington: Secretaria General de la Organización de los Estados Americanos, 1978. $114 \mathrm{p}$.

19. SANTOS, G. P.; ZANUNCIO, T. V.; ZANUNCIO, J. C. Desenvolvimento de Thyrinteina arnobia Stoll (ípterara: Geometridae) em folhas de Eucalyptus urophylla e Psidium guajava. Anais da Sociedade Entomológica do Brasil, v. 29 , n. 1, p. 13-22, 2000

20. SANTOS, H. J. G. et al. Interação de Metarhizium anisopliae (Metsch) Sorok., Beauveria bassiana (Bals.) Vuill. E o parasitóide Oomyzus sokolowkii (Kurdjumov) (Hymenoptera: Eulophidae) sobre larvas da traça-das-crucíferas, Plutella xylostella (L) (ípterara: Plutellidade). Neotropical Entomology, v. 35, n. 2, p. 241-245, 2006.

21. TORRES, J. B.; ZANUNCIO, J. C.; MOURA, M. A. The predatory stinkbug Podisus nigrispinus: biology, ecology and augmentative releases for lepidoperan larval control in Eucalyptus forests in Brazil. Perspective In Agriculture Veterinary Science Nutrition And Natural Resources, v. 15, n. 1, p. 1-18, 2006.

22. WITTMEYER, J. L.; COUDRON, T. A. Life table parameters, reproductive rate, intrinsic rate of increase, and estimated cost of rearing Podisus maculiventris (Heteroptera: Pentatomidae) on an artificial diet. Journal of Economic Entomology, v. 94, n. 6, p. 1344-1352, 2001.

23. ZANUNCIO, J. C. et al. Alguns aspectos da biologia de Dirphia rosacordis (Lepidoptera: Saturnidae) em folhas de eucalipto. Revista Árvore, v. 36, n. 1, p. 112-117, 1992.

24. ZANUNCIO, J. C. et al. Nova metodologia para criação em laboratório de hemípteros predadores. Revista Ceres, v. 41 , n. 1, p. 88-93, 1994

25. ZANUNCIO, J. C. et al. Nymphal development and reproduction of Podisus nigrispinus (Heteroptera: Pentatomidae) fed with combinations of Tenebrio molitor (Coleoptera: Tenebrionidae) pupae and Musca domestica (Diptera: Muscidae) larvae. Biocontrol Science and Technology, v. 11, n. 3, p. 331-337, 2001.

26. ZANUNCIO, T. V. et al. Utilização de mudas de "Eucalyptus grandis" melhora a criação do predador "Brontocoris tabidus" (Het.: Pentatomidae) em laboratório. Arquivos do Instituto Biológico, v. 75, n. 2, p. 167-172, 2008.

Recebido em 06/11/2008

Aceito em 10/08/2009 